\title{
Reference frequencies comb generation for microwave photonics ADC with signal spectral-interval estimation
}

\author{
Victor Kulagin ${ }^{1,2,3, *}$, Vladimir Cherepenin ${ }^{2}$, Sergey Kontorov ${ }^{4}$, Denis Prokhorov ${ }^{3}$, \\ and Victor Valuev ${ }^{2,3}$ \\ ${ }^{1}$ Sternberg Astronomical Institute of Lomonosov Moscow State University, 119992, Moscow, Russia \\ ${ }^{2}$ Kotel'nikov Institute of Radio-engineering and Electronics of RAS, 125009, Moscow, Russia \\ ${ }^{3}$ National Research Nuclear University MEPhI, 115409, Moscow, Russia \\ ${ }^{4}$ Skolkovo Institute of Science and Technology, 121205, Moscow region, Russia
}

\begin{abstract}
Formation of a reference frequency comb in a circuit comprising a highly stable continuous laser and an amplitude MachZehnder modulator with two independent RF inputs working with large modulation index for use in a high-speed microwave photonics ADC with spectral-interval estimation is investigated by numerical simulation. In such ADCs, the entire spectrum of the input signal is divided by microwave photonics elements into spectral intervals, each of which uses a microwave photonics converter to transform the signal to an intermediate frequency (for each part of the spectrum of the microwave input signal, its own reference frequency is used for heterodyning). The requirements for a multimode source of optical reference frequencies for high-speed microwave photonics ADC are formulated. To study the characteristics of such a source, numerical modeling is carried out. It is shown that the frequency noise of the laser can be partially suppressed due to their compensation when converted to an intermediate frequency. Experimental study of the reference frequency comb generator layout showed a good agreement of the obtained characteristics with the results of numerical simulation.
\end{abstract}

\section{Introduction}

Analog-to-digital conversion (ADC) is a fundamental procedure in the rapidly developing information technologies used in many fields of science and techniques. Modern problems of information processing require high speed (bandwidth) and resolution of the ADC. In the last decade, new methods of microwave signal reception, processing and analysis implemented on the basis of microwave photonics have been rapidly developing [1-3]. The obvious advantages of microwave photonics devices are an extremely wide frequency band, which can be 100 gigahertz or more, an ability to software control modes of operation, flexibility of the formation of new devices that perform certain functions, protection from electromagnetic interference, good mass-dimensional characteristics, etc.

* Corresponding author: victorvkulagin@yandex.ru 
In the currently developed high-speed photonics ADCs, each adjacent time readout is usually processed by its own channel [4], and the full output signal must be obtained as a result of the joint processing of all samples, which requires parallel processing of information. At the same time, for input signals with a given bandwidth, part of the data received after processing will not contain useful information and will be discarded, and this part is the greater the higher the ratio of the carrier frequency to the bandwidth of the signal, i.e. this method of digitizing the microwave signal is redundant. An approach based on the microwave photonics spectral-interval signal conversion [5] can be more effective for the input microwave signal with a given bandwidth, which can also be successfully applied to signals with a spectrum width of the order of the maximum frequency.

\section{Microwave photonics ADC with spectral-interval estimation of the input signal}

Microwave photonics high-speed ADC with a spectral interval estimation of the input signal (Fig. 1) is based on the use of a multichannel optical heterodyning (spectral-interval estimation) [5]. Here, the input microwave signal using microwave photonics elements is divided into a number of spectral intervals (channels) containing useful information. Next, each channel uses a microwave photonics converter to pre-process the signal and transform it to an intermediate frequency (for every channel, i.e., for each part of the spectrum of the microwave input signal, its own reference frequency is used for heterodyning), and then the signal is digitized by a commercial electronic ADC. Thus, the whole system consists of a set of identical in structure receiving channels, each of which operates in its frequency range, on which the entire spectrum of the input signal is divided. In many cases, the output signals of these channels are already sufficient to obtain the necessary information, i.e. joint processing of all output signals may not be required at all.

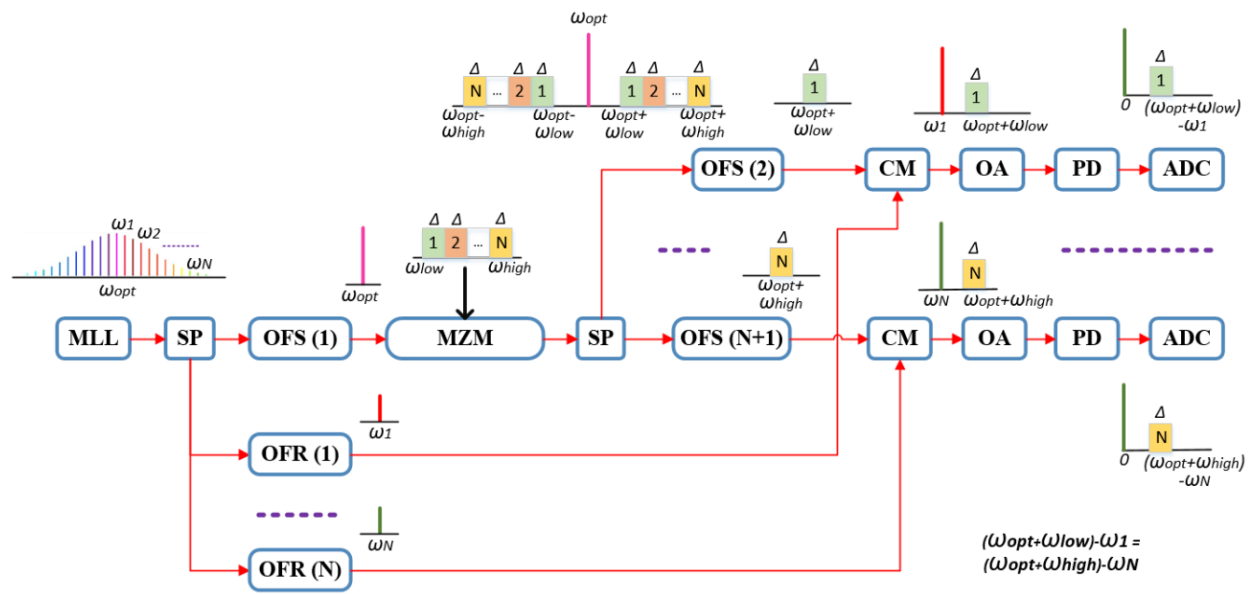

Fig. 1. Block diagram of the photonic ADC based on a mode-locked laser: MLL - mode-locked laser, SP - splitters, OFS - optical signal filters, OFR - optical reference mode filters, MZM - amplitude Mach-Zehnder modulators, CM - combiners, OA - optical amplifiers, PD - photodetectors, ADC electronic analog-to-digital converters, $\mathrm{CWL}$ - continuous wave laser, $\omega_{\text {low }}-$ lowest frequency of the broadband signal, $\omega_{\text {high }}-$ highest frequency of the broadband signal, $\omega_{\text {opt }}-$ MLL mode for signal modulation, $\omega_{1}-$ MLL mode next to $\omega_{\mathrm{opt}}, \Delta$ - part of a bandwidth of a broadband signal.

If it is necessary to restore the broadband input signal in digital form from the output signals of several channels, coherent signal processing in each channel with an accurate time reference should be provided, which assumes small nonlinearities of the conversion and 
preservation of phase information. In this case, the restoration of the full input microwave signal from the output signals of several channels can be provided by the following procedure. At the output of each channel, a full spectrum is formed, including the phase component, of the part of the input signal that falls into the spectral interval of the channel. Then, by a required number of samples of the spectrum, it is possible to completely restore the original signal in the time domain. Thus, choosing the desired sequence from a set of individual spectra in intervals, it is possible to obtain the full input signal in digital form using computer processing [5]. Overlapping sections of the spectra can be used to calibrate the transfer characteristics of neighboring channels and data correction.

\section{Generation of the optical reference frequency comb}

An important element of a high-speed microwave photonics ADC with spectral-interval signal estimation is a device for generating a comb of reference frequencies. The stability of these frequencies determines the characteristics of the microwave photonics converters to the intermediate frequency in the various channels and, ultimately, the effective number of bits of the ADC itself. Usually, a stable pulsed laser with mode synchronization is the basis of such systems. In the frequency domain, the oscillation spectrum of such lasers is a set of equidistant modes with different frequencies, which can be separated by a comb of optical filters. At the same time, multimode optical lasers with synchronized modes are not stable enough, expensive, and have a long time to enter the operating mode, in addition, the possibility of their integrated optical implementation is significantly complicated. An additional disadvantage of their use in high-speed microwave photonics ADC with spectralinterval estimation is generation of too many modes (from several thousand to tens of thousands) and a significant inequality of their amplitudes.

To use a multimode optical reference frequency source in a high-speed microwave photonics ADC based on spectral-interval estimation of the input signal, the following conditions must be met. The distance between the lines should be of the order of $1-2 \mathrm{GHz}$, which is determined by the speed of available electronic ADCs used to digitize the output signals from a multi-channel microwave photonics frequency converter. The total number of formed lines should be approximately equal to the required number of channels. Low source phase noise is extremely important because it directly determines the final characteristics of the ADC. It is possible to combine all these requirements in a scheme that includes a highly stable continuous laser and a frequency comb formation system that includes several amplitude and phase modulators with a large modulation index to generate a sufficient number of sideband lines. In the simplest case, only one amplitude modulator with two independent RF inputs with the same frequency can be used [6]. Selection of the necessary lines from the frequency comb will be carried out by narrow-band optical filters. The advantage of such systems is the ability to independently set the average frequency of the comb, determined by the laser frequency, and the distance between the lines in it, which is determined by the modulation frequency. The noise characteristics of the comb depend mainly on the noise structure of the modulating RF signal and the characteristics of the continuous laser. The use of an optoelectronic oscillator to generate a modulating RF signal will significantly reduce the phase noise of the entire system. The advantage of such systems also is the ability to generate combs with a given number of frequency lines, which significantly increases the energy efficiency of the ADC.

\section{Numerical simulation of the reference frequency comb generator}

A numerical study of the characteristics of the optical frequency comb was carried out for a circuit based on an amplitude modulator with two independent inputs [6]. Possible sources 
of noise and fluctuations in the system and their characteristics were found, a mathematical model was created and a numerical code was developed using the MATLAB software package that allows analyzing the main characteristics of the mode comb generator.

Spectral density of the modulator output field is shown in Fig. 2. The modulating frequency is $1 \mathrm{GHz}$, modulation indices in the arms are 2 and 3.5. The red ellipse highlights 7 central lines with inequality of amplitudes less than $3 \mathrm{~dB}$. The optical frequency is chosen to be $10^{12} \mathrm{~Hz}$ due to the limited computing power of the servers.

a)

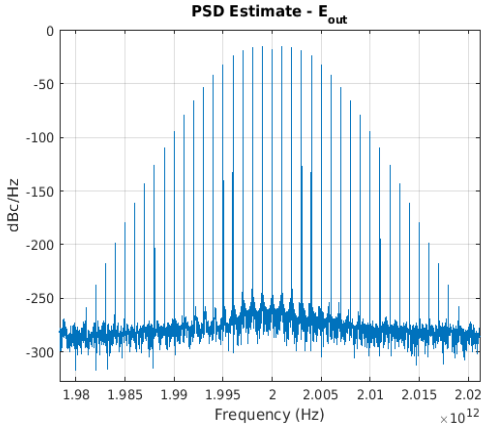

b)

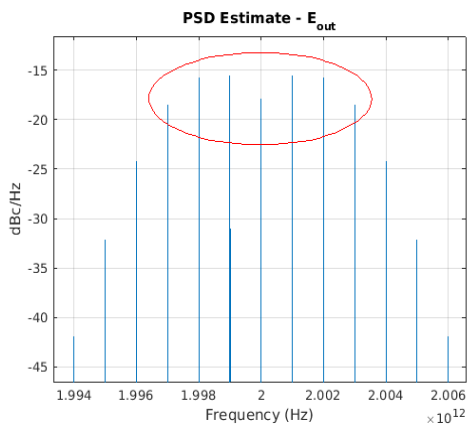

Fig. 2. Spectral density of the modulator output field for $1 \mathrm{GHz}$ modulating frequency: full view (a) and zoom of the central lines (b). Modulation indices in the arms are 2 and 3.5. The red ellipse highlights the central lines with inequality of amplitudes less than $3 \mathrm{~dB}$ ( 7 lines in total). The optical frequency is chosen to be $10^{12} \mathrm{~Hz}$ due to the limited computing power of the servers.

For high modulation index, the spectral density of the modulator output field is shown in Fig. 3. Here, modulation indices in the arms are 15 and 16.5. The red ellipse represents the center lines with amplitude irregularity less than $3 \mathrm{~dB}$ (25 modes), and the green ellipse represents modes with irregularity less than $6 \mathrm{~dB}$ (33 modes).

a)

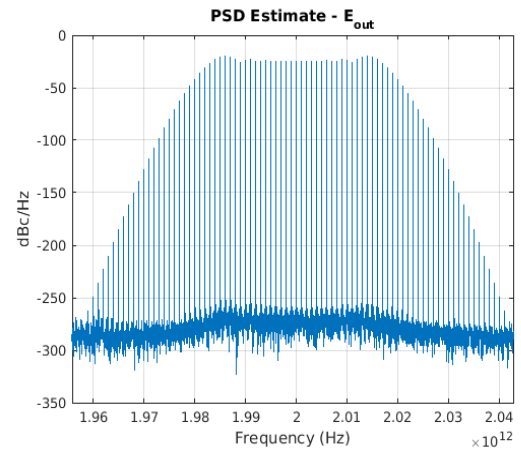

b)

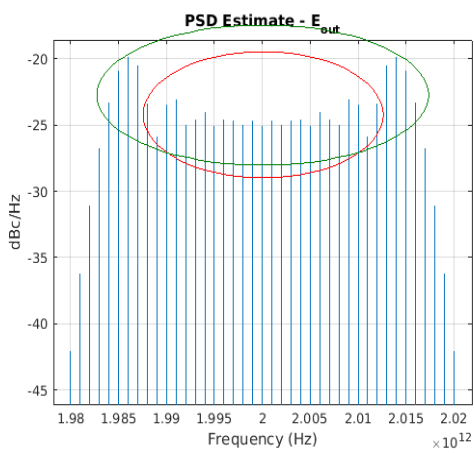

Fig. 3. Spectral density of the modulator output field for $1 \mathrm{GHz}$ modulating frequency: full view (a) and zoom of the central lines (b). Modulation indices in the arms are 15 and 16.5. The red ellipse represents the center lines with amplitude inequality less than $3 \mathrm{~dB}$ ( 25 modes), and the green ellipse represents modes with inequality less than $6 \mathrm{~dB}$ (33 modes).

Noise characteristics of the generated comb can be investigated through selecting two modes with optical filters, combining them at a photodetector and then measuring the signal at the difference frequency with a spectrum analyzer. We simulated this process numerically using the $10 \mathrm{GHz}$ frequency as the modulation frequency for comb generation. Spectral density of the signal at the output of the photodetector is shown in Fig. 4. When two adjacent modes are isolated from the comb and combined at a photodetector the signal with frequency $10 \mathrm{GHz}$ can be obtained (Fig. 4a). In Fig. 4b, the signal with frequency 30 $\mathrm{GHz}$ are shown when selecting two modes with a frequency difference of $30 \mathrm{GHz}$ from the 
comb. The laser linewidth here is $500 \mathrm{kHz}$ and the laser power is $40 \mathrm{~mW}$. Fig. 4 shows that for good parameters of the laser, noises can be small at the output of the photodetector.

a)

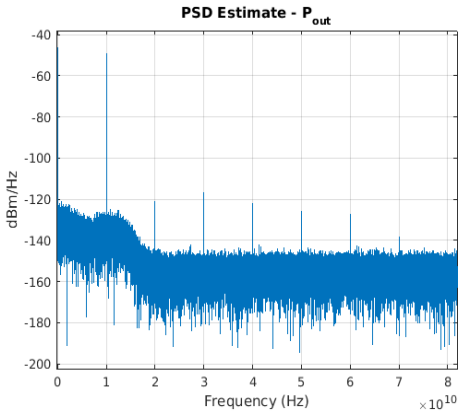

b)

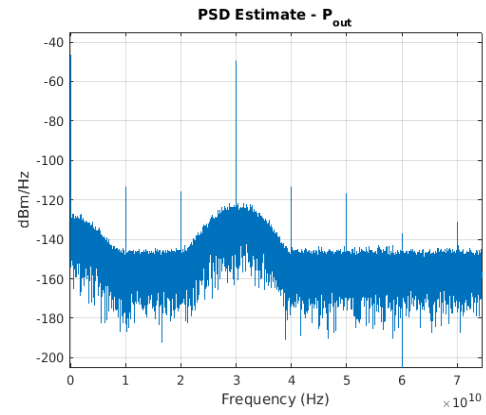

Fig. 4. Spectral density of the signal at the output of the photodetector: signal with frequency $10 \mathrm{GHz}$ when two adjacent modes are separated from the comb (a) and signal with frequency $30 \mathrm{GHz}$ when selecting from the comb two modes with a frequency difference of $30 \mathrm{GHz}(\mathrm{b})$. The laser line width is $500 \mathrm{kHz}$, the laser power is $40 \mathrm{~mW}$.

Same simulations with one optical mode modulated by the RF signal are shown in Figs. 5 and 6 . The modulation frequency of the signal was $100 \mathrm{MHz}$, which is considerably larger than the laser linewidth.

a)

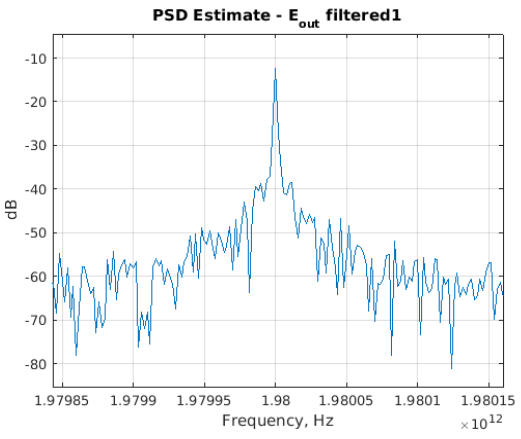

b)

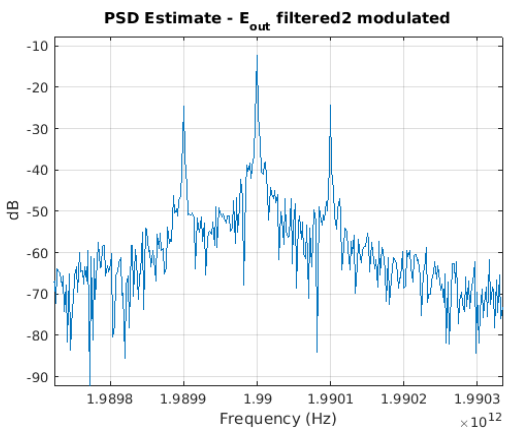

Fig. 5. Spectral densities of two optical modes used to form a modulated microwave signal: mode without modulation (a) and mode with amplitude modulation with frequency $100 \mathrm{MHz}$ (b).

Spectral density of the signal at the output of the photodetector during formation of modulated microwave signal is shown in Fig. 6. This figure proves that the modulation signal can be easily transferred to the mode with the difference frequency.

To check the stability of the system, we simulated also the case when the laser linewidth is considerably larger than the modulation frequency of the signal. Spectral density of the signal at the output of the photodetector for this case is presented in Fig. 7. Modulation lines are not visible at all on the spectral density of the modulated optical mode (Fig. 7a), but after the photodetector they are clearly distinguishable (Fig. 7b). Their height with respect to noises is reduced by $15 \mathrm{~dB}$ due to large laser frequency noises. In this case, laser wavelength was $5 \mathrm{GHz}$ which is considerably larger than $100 \mathrm{MHz}$ used for modulation. So this system can be stable against the laser frequency noises.

\section{Practical schemes for realization of the reference frequency comb generator}

Frequency comb generator based on a continuous laser and studied above can be realized when the following conditions are met. The voltage required to form considered combs is determined by the half-wave voltage of the modulator. Thus, for a half-wave voltage equal 
to $5 \mathrm{~V}$, the maximum modulation voltage should be about $5.5 \mathrm{~V}$ to form 7 lines and about $25 \mathrm{~V}$ to form 33 lines. The driver power for the modulator is about $0.6 \mathrm{~W}$ in the first case and about $13 \mathrm{~W}$ in the second. The use of more sophisticated circuits with a larger number of elements can reduce this power several times.

a)

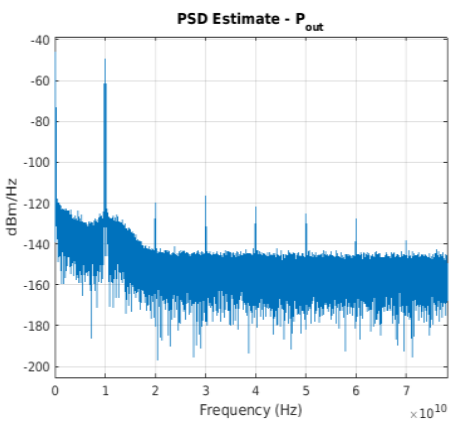

b)

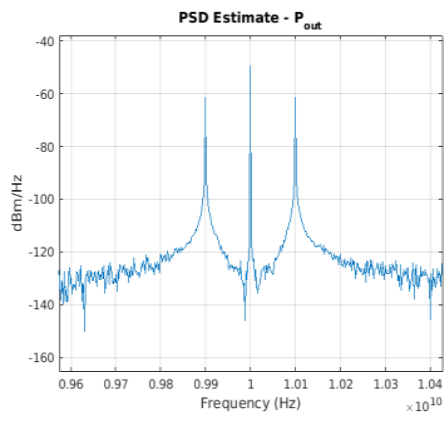

Fig. 6. Spectral density of the signal at the output of the photodetector during formation of modulated microwave signal: general view (a) and zoom view of the main mode (b).

a)

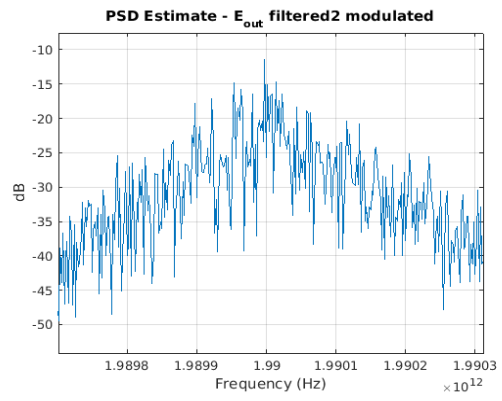

b)

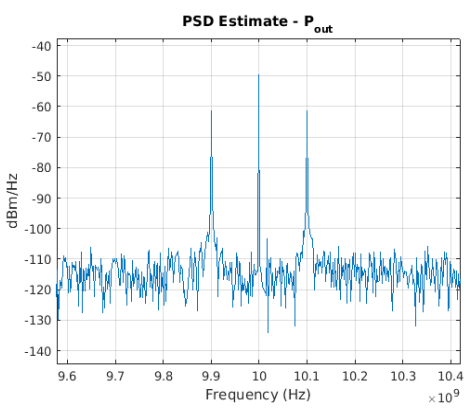

Fig. 7. Spectral density of the optical mode with amplitude modulation (a) and the output signal of the photodetector (b) in the formation of a modulated microwave signal using a laser with a linewidth much larger that the modulation frequency.

One of these schemes is based on several amplitude modulators working sequentially [7]. These modulators have different modulation frequencies and indices which are chosen to sequentially fill the gaps between the previously formed modes. The advantage of this scheme is the ability to work with a smaller modulation index. However, it requires several RF sources with different frequencies that can be inconvenient and can increase the final noises of the generated comb.

Only three modulation frequencies are required to form 20 mode lines with two amplitude modulators and one double parallel modulator [8]. Again, ordinary MachZehnder modulators form 4 lines with very small amplitude inequality and the gaps between these lines are filled with double parallel modulator. In these scheme, ordinary modulation indices are used and generated comb has very high quality.

Formation of five or seven lines can be realized in double parallel modulator alone using one RF modulation source only [9]. It can be enough for ADC with maximal frequency about $10 \mathrm{GHz}$. The RF modulating signal is divided between two arms of double parallel modulator unequally and the division ratio defines the number of lines generated. This scheme has small amplitude inequality of the lines.

\section{Experimental results}

Experimental testing of the approach was realized using scheme based on double parallel modulator with one RF modulation source [9]. We used two tunable optical filters to select 
required modes. Then, one optical mode can be RF modulated (or not) and then, two modes combined and the sum of the fields photodetected. The characteristics of generated lines were investigated using oscilloscope and RF spectrum analyzer. Laser power was $16 \mathrm{dBm}$. For modulation of the laser field, $6 \mathrm{GHz}$ microwave signal was used so the comb had 5 lines with $6 \mathrm{GHz}$ distance between them. Tunable optical filters had the bandwidth $2 \mathrm{GHz}$ which defines the modulation frequency. The spectral density of the selected modes registered with an optical spectrum analyzer is shown in Fig. 8a, and difference mode oscilloscope signal and spectral density is presented in Figs. 8b and 8c. Also, we measured the difference mode generation in the presence of RF signal modulation of one optical mode. These figures confirm the simulation results presented earlier.
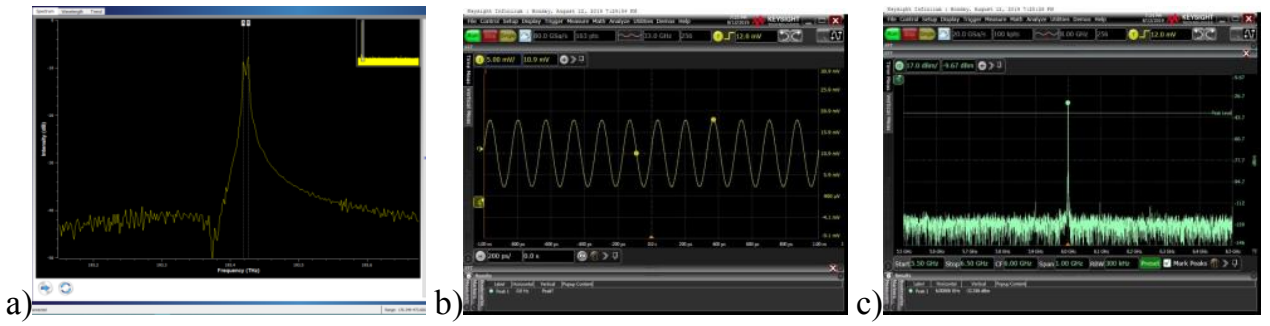

Fig. 8. Spectral density of the selected modes registered with an optical spectrum analyzer (a), and difference mode oscilloscope signal (b) and spectral density (c).

\section{Conclusion}

High-speed microwave photonics ADCs with spectral-interval estimation are more effective for input signals with a limited bandwidth than photonic ADCs with processing a sequence of time samples by different channels. The formation of a reference frequency comb for ADC with spectral-interval estimation in a circuit comprising a highly stable continuous laser and an amplitude modulator with two independent RF inputs is numerically investigated. The requirements to the multimode optical source of reference frequencies for high-speed microwave photonics ADCs are formulated, in particular, the distance between the lines should be of the order of 1-2 GHz, and the total number of generated lines is approximately equal to the desired number of channels, besides, comb source should have low noises. It is shown that the frequency noises of the laser can be partially suppressed due to their compensation when converted to an intermediate frequency. Experimental study of the reference frequency comb generator layout showed a good agreement of the obtained characteristics with the results of numerical simulation.

This work was supported by the Russian Foundation for Basic Research, project 17-07-01411a. The authors are grateful to R.S. Starikov, E.Y. Zlokazov, D.S. Zemtsov, I. Zh. Hafizov and A.V. Nebavsky for the help with experimental measurements.

\section{References}

1. J. Capmany, D. Novak, Nat. Photonics, 1, 319 (2007)

2. A.J. Seeds, K.J. Williams, J. Lightwave Technol., 24, 4628 (2006)

3. J.P. Yao, J. Lightwave Technol., 27, 314 (2009)

4. G.C. Valley, Optics Express, 15, 1955 (2007)

5. V.A. Cherepenin, S.M. Kontorov, V.V. Kulagin, D.A. Prokhorov, A.N. Shulunov, V.V. Valuev, IEEE Conference Publications: 48th European Microwave Conference (EuMC), 796 (2018) 
6. T. Sakamoto et. al, Optics Letters, 32, 1515 (2007)

7. T. Sakamoto et al, Light, Energy and the Environment Congress, OSA 2016, FTh3B.3 (2016)

8. X. Zhou et al, Optics Communications, 313, 356, (2014)

9. L. Shang et al, Optics Communications, 356, 70 (2015) 